\title{
Perfil epidemiológico das doenças sexualmente transmissíveis notificadas pelo Sistema de Informação de Agravos de Notificação no sul da Bahia
}

\author{
Semírames de Oliveira Bittencourt*, Michelle Araújo Moreira, D.Sc.**
}

${ }^{*}$ Graduada em Enfermagem pela Universidade Estadual de Santa Cruz (UESC), Ilhéus/BA, **Enfermeira, Sanitarista, Professora Adjunta de Saúde da Mulher da Universidade Estadual de Santa Cruz-UESC, Departamento de Ciências da Saúde, Orientadora da pesquisa

\begin{abstract}
Resumo
Introdução: As Doenças Sexualmente Transmissíveis (DST) são a segunda maior causa mundial de adoecimento entre mulheres com cerca de 10 milhōes de casos/ano. Objetivo: Traçar o perfil epidemiológico das DST notificadas pelo Sistema de Informação de Agravos de Notificação (SINAN) na cidade de Itabuna/BA. Material e métodos: Estudo quantitativo, descritivo e exploratório com coleta de dados no SINAN, na série temporal de 2007 a 2011 . Resultados: Verificou-se que a média de notificações foi de 27,2 casos para AIDS, 131,2 para hepatite e de 13 para sífilis gestacional. A proporção de casos permanece predominante no sexo masculino, apesar do aumento na população feminina, sugerindo o fenômeno da feminização. Em relação à faixa etária, perceberam-se maior número de casos entre 20 e 29 anos (AIDS), 30 a 39 anos (Hepatite), 20 a 29 anos (Sífilis). Quanto à escolaridade, identificou-se que os sujeitos com ensino fundamental estáo entre os mais acometidos. No que tange à cor/etnia, nota-se maior número de negros em relaçáo aos brancos. Conclusão: Identificou-se um número expressivo de DST entre jovens, homens e mulheres, negros e com ensino básico. Nota-se que o processo de subnotificaçáo dificulta a análise do perfil epidemiológico, o que interfere no planejamento local de ações de controle e prevenção.
\end{abstract}

Palavras-chave: doenças sexualmente transmissíveis, perfil de saúde, notificação de doenças, saúde da mulher.

\section{Abstract \\ Epidemiological profile of sexually transmitted diseases notified by Notifiable Disease Information System in southern Bahia}

Introduction: Sexually Transmitted Diseases (STD) are the second leading cause of illness among women worldwide with approximately 10 million cases per year. Aim: To describe the epidemiology profile of STD reported by the Notifiable Disease Information System (SINAN) at Itabuna, Bahia. Methods: This was a quantitative, descriptive and exploratory study with data collection in SINAN in time series from 2007 to 2011. Results: Was verified that the average number 
of notifications was 27.2 cases for AIDS, 131.2 for hepatitis and 13 for gestational syphilis. There is a predominance of male cases, although the increase in the female population, suggesting the phenomenon of feminization. In relation to age group, was pointed a higher number of AIDS cases between adults 20-29 years old, 30-39 years old (Hepatitis), 20-29 years old (Syphilis). Regarding education, was observed that patients with primary education are among the most affected. Regarding the color/ethnicity, there are more African-american people infected than white people. Conclusion: Was identified a significant number of STDs among young men and women, African-american and with primary education. The sub-notification process causes difficulties in analyzing the epidemiological profile, which interferes with the local planning actions of controlling and prevention.

Key-words: sexually transmitted diseases, health profile, disease notification, women's health.

\section{Resumen}

\section{Perfil epidemiológico de enfermedades de transmisión sexual notificadas por el Sistema de Información sobre Enfermedades de Declaración Obligatoria en la microregión del sur de Bahia}

Introducción: Las Enfermedades de Transmisión Sexual (ETS) representan un problema de salud pública en Brasil, porque es la segunda mayor causa de enfermedades entre mujeres en todo el mundo con aproximadamente 10 millones de casos/ años. Objetivo: Describir el perfil epidemiológico de las ETS reportados por el Sistema de Información sobre Enfermedades de Declaración Obligatoria (SINAN) en la ciudad de Itabuna, Bahía. Material e métodos: Estudio cuantitativo, descriptivo y exploratorio que utilizó la colecta de datos en el SINAN, en la serie temporal de 2007 a 2011. Resultados: Se verificó un promedio de notificaciones de 27,2 casos del SIDA, 131,2 de hepatitis y de 13 de sífilis gestacional. La proporción de casos sigue siendo predominante en los varones respecto a las mujeres, a pesar del aumento en la población femenina, lo que sugiere el fenómeno de la feminización. En relación al grupo de edad, se registró un mayor número de casos de SIDA entre los 20 y los 29 años, de hepatitis entre 30 y 39 años y de sífilis entre 20 y 29 años. En cuanto a la educación, se identificó que los sujetos con educación primaria se encuentran entre los más afectados. Con respecto al color/etnia, había mayor número de negros infectados en relación a los blancos. Conclusión: Se identificaron un número significativo de ETS entre jóvenes, hombres y mujeres, negros y con educación básica. Se observa que el proceso de subnotificación permite dificultades en el análisis del perfil epidemiológico, que interfiere en la planificación de acciones locales de control y prevención.

Palabras-clave: enfermedades de transmisión sexual, perfil de salud, notificación de enfermedad, salud de las mujer.

\section{Introdução}

As Doenças Sexualmente Transmissíveis (DST) estão entre os problemas de saúde pública mais frequentes em todo o mundo. Estes agravos por provocarem inflamação e/ou ulceração genital da pele e mucosas facilitam a infecção pelo Vírus da Imunodeficiência Humana (HIV), resultando em complicaçóes que podem levar à esterilidade feminina e masculina, infecçóes congênitas, aborto, nascimento de bebês prematuros, deficiência física ou mental, alguns tipos de câncer e até a morte [1-3].

As DST representam a segunda maior causa mundial de adoecimento entre as mulheres de 15 a 49 anos, sendo que a cada ano surgem 333 milhóes de casos novos. No Brasil, a avaliação epidemiológica torna-se ineficiente em virtude da subnotificação de casos confirmados. Percebe-se que o Brasil tem uma baixa implementação na notificação compulsória, além de um atendimento deficitário aos usuários com DST, o que torna o número de casos notificados abaixo da estimativa da Organização Mundial de Saúde (OMS), algo em torno de 200 mil casos ao ano. Por esta razão, os dados relacionados à incidência de DST são parcos, salvo em relação à Síndrome da Imunodeficiência Adquirida (AIDS) e sífilis $[3,4]$.

Em relação ao HIV, a epidemia apresenta um aumento no número de casos entre os heterossexuais, mulheres, jovens, pessoas com baixa escolaridade e renda, sugerindo o fenômeno da feminização, pauperização e interiorização do agravo. Assim, o Boletim Epidemiológico de 2011 destaca que "em 1989, a razão de sexos era de cerca de seis casos de AIDS no sexo masculino para cada caso no sexo feminino. Em 2010, chegou a 1,7 casos em homens 
para cada um em mulheres". Dentro desta população, destacam-se mulheres em união consensual ou casadas com muito tempo de relacionamento afetivo e sexual [4-6].

Em virtude do cenário apresentado, sabe-se que as questóes de gênero são aspectos preponderantes para entender as relaçóes entre os sujeitos, como estas influenciam nas relaçóes de poder estabelecidas entre homens e mulheres no meio social, bem como na sexualidade e vulnerabilidade feminina às DST. Para tanto, a vulnerabilidade pode ser entendida como:

(...) o resultado de uma conexão de fatores que podem ser individuais (biológicos, cognitivos e comportamentais), culturais (submissão a padrōes e crenças morais, hierarquias, relaçôes de poder, questôes de gênero) e sociais (relacionados às questôes econômicas e sociais), dependentes entre si e mutuamente influenciáveis, assumindo pesos e significados diversos que variam no decorrer do tempo e determinam o grau de susceptibilidade de indivíduos e grupos em relação a questôes de saúde (...) [7].

Dessa forma, as relaçóes desiguais entre homens e mulheres são observadas nos diferentes meios políticos, culturais e socioeconômicos, tendo expressão no âmbito das famílias e parcerias, sociedade e culturas, estas organizadas em sistemas de crenças, códigos e valores, atuando conjuntamente como fatores para a vulnerabilidade das mulheres às DST [8-10].

Com relação à vulnerabilidade sexual nota-se que esta permanece atrelada às relaçôes entre as parcerias. $\mathrm{O}$ aparecimento das DST pode desestruturar os relacionamentos pela instabilidade na relação de confiança entre os pares. Além disso, na maioria das vezes, quando um parceiro negocia o uso do preservativo são levantadas desconfianças no que tange à fidelidade. Essas questóes dificultam o desenvolvimento de estratégias de prevenção às DST, especialmente para pessoas que vivem relacionamentos estáveis. A inexistência de diálogo sobre sexualidade entre os casais torna difícil qualquer adoção de medidas preventivas e, muitas vezes, este diálogo só ocorre em momentos de tensão, como é o caso da presença de uma DST. Portanto a suscetibilidade às DST aumentou consideravelmente ao longo dos anos devido ao comportamento sexual desprotegido, creditando apenas o fator confiança nas parcerias $[11,9]$.

No que tange à suscetibilidade relacionada ao fator idade, nota-se que a AIDS é incidente em ambos os sexos, preferencialmente na faixa etária de 25 a 49 anos. Contudo, evidencia-se um aumento gradativo dos casos entre jovens de 13 a 19 anos. Essa faixa etária apresenta um grande número de casos de AIDS, sobretudo nas mulheres. Isso ocorre em decorrência da atividade sexual precoce, multiplicidade de parceiros sexuais, modificaçóes físicas e psíquicas típicas da adolescência, caracterizando-a como um período marcado por intensas transformaçóes no comportamento sexual.

Os jovens vivenciam etapas conflituosas no comportamento sexual e passam por diversas influências sociais, culturais, familiares e midiáticas. Essas influências repercutem diretamente nas suas atitudes com o próprio corpo e com os agravos que dele derivam. Nesta fase, percebe-se grande vulnerabilidade física, psicológica e social. A vulnerabilidade pode ser entendida como a junção dos diferentes fatores e está na dependência da capacidade de reinterpretar criticamente os estímulos sociais de perigo e/ou risco $[3,5,12,13]$.

Focando nas questóes étnicas e raciais, os dados confirmam que a proporção de casos de AIDS no ano de 2009 foi predominante na raça branca $(47,7 \%)$ seguida da cor parda $(35,8 \%)$ e preta $(11,0 \%)$. Nota-se que há uma prevalência equiparada entre brancos e negros, visto que a cor parda é enquadrada atualmente dentro da etnia negra pelo Instituto Brasileiro de Geografia e Estatística (IBGE) [14].

Quanto à escolaridade, em 2009, 25,1\% do total de indivíduos notificados para AIDS possuíam entre quatro e sete anos de estudo, 30,0\% entre 8 e 11 anos e 8,8\% entre um a três anos. Observa-se uma tendência no aumento do número de casos de AIDS em pessoas com nível de escolaridade mais elevado, revelando dificuldades no uso do preservativo mesmo com acesso às informaçóes [14].

Considerando o número ampliado de casos nacionais e a subnotificação das DST associado à importância do SINAN na elaboração de propostas gestoras para minimizar a vulnerabilidade sexual dos sujeitos, justifica-se a necessidade do estudo. Este se propôs a traçar um perfil epidemiológico das DST notificadas em um município do interior da Bahia, fornecendo subsídios para que gestores, profissionais da saúde e comunidade envolvida possam pensar em estratégias de cuidado ampliado. 
Além disso, a definição do perfil epidemiológico das DST mais prevalentes na cidade de Itabuna-Bahia contribuirá para que profissionais da saúde e de áreas correlatas possam desenvolver açóes adaptadas ao padrão social, econômico, cultural, típico desta localidade, garantindo a elaboração de outros estudos que possam discutir a vulnerabilidade sexual.

Diante do apresentado, definiu-se como objetivo geral: traçar o perfil epidemiológico das DST notificadas pelo SINAN na cidade de Itabuna-Bahia segundo variáveis de sexo, faixa etária, escolaridade e cor/etnia.

Para isto, tive como objetivos específicos:

- Levantar as DST notificadas pelo SINAN na cidade de Itabuna-Bahia.

- Discutir a ocorrência das DST notificadas pelo SINAN na cidade de Itabuna e a sua relaçáo com a vulnerabilidade sexual.

\section{Material e métodos}

Trata-se de um estudo quantitativo, descritivo e exploratório. A abordagem quantitativa compreende o estudo do comportamento de variáveis individuais ou de maneira associativa [15]. Este autor acredita que a perspectiva quantitativa permita o entendimento de um objeto, garantindo um grau de validez externa e interna e, dessa forma, proponha programas de intervenção concretos e adequados às particularidades de todos os setores sociais que se pretende atingir.

A perspectiva descritiva tem por finalidade principal conhecer as características de um determinado fenômeno para procurar, posteriormente, explicaçóes das causas e consequências do mesmo [16].

O lócus do estudo foi o município de Itabuna, situada no Estado da Bahia. Este município possui uma população total estimada em 204.110 habitantes e uma população urbana de 191.694 pessoas. A gestão em saúde define-se pela gestão plena da atençáo básica ampliada, contando com os serviços da atenção primária em 32 unidades, das quais 21 correspondem às Estratégias de Saúde da Família (ESF).

Para a coleta foram utilizados os dados do Sistema de Informação de Agravos de Notificação (SINAN) referentes às DST notificadas na cidade de Itabuna-Bahia a exemplo da AIDS, Hepatite B e Sífilis Gestacional, na série temporal de 2007 a 2011, levantado na Vigilância Epidemiológica Municipal. Convém destacar que foram respeitados os aspectos éticos, respaldada na Lei no 9.610 de 19 de fevereiro de 1998 que trata dos direitos autorais [17]. Salienta-se que não houve necessidade de submissão do projeto a um Comitê de Ética em Pesquisa por tratar-se de estudo descritivo com utilizaçáo de dados secundários proveniente do sistema nacional de notificação de agravos.

Para ampliar a fundamentaçáo teórica, realizou-se um levantamento bibliográfico sobre a temática nas bases de dados Scientific Eletronic Library On line (Scielo), Literatura Latino-Americana e do Caribe em Ciências da Saúde (Lilacs), Biblioteca Virtual de Saúde (BVS) em uma série temporal de 2006 a 2011, utilizando como língua oficial o português e os descritores em saúde: vulnerabilidade sexual, DST, perfil epidemiológico, gênero. Além disso, utilizaram-se livros e manuais sem definição histórica.

Os dados quantitativos oriundos do SINAN foram tratados estatisticamente por proporçóes com a devida apresentação em gráficos a seguir.

\section{Resultados e discussão}

As principais DST registradas no município de Itabuna e classificadas como de notificação compulsória, no período de 2007 a 2011, foram a AIDS, Hepatite B e Sífilis gestacional, tratadas segundo variáveis de sexo, faixa etária, escolaridade e etnia, apresentadas abaixo em ordem sequencial.

\section{AIDS}

Gráfico 1 - Proporção de casos de AIDS por sexo, Itabuna/BA, 2007 a 2010.

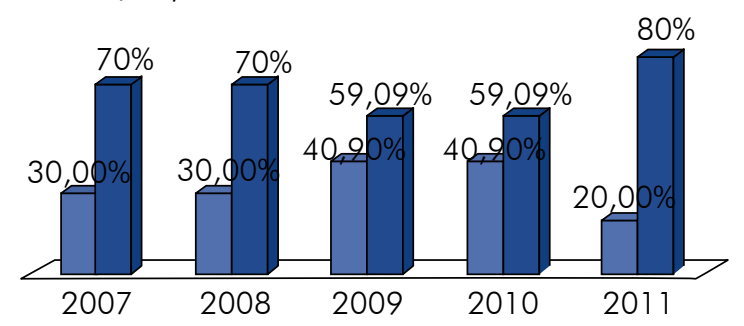

De acordo com o Gráfico 1, houve predomínio de $70 \%$ em homens e $30 \%$ em mulheres nos anos de 2007 e 2008, sendo que nos anos de 2009 e 2010 manteve-se um equilíbrio entre homens e mulheres atingindo 59,09\% e 40,90\%, respectivamente. No ano de 2011, há um retorno 
no número ampliado de casos em homens $(80 \%)$ em comparaçáo às mulheres $(20 \%)$, o que reflete que a proporção mantém-se preferencialmente no sexo masculino. Este aumento decorre das crenças e tabus que permeiam o uso do preservativo e que acabam acarretando em descuido nas condutas de autocuidado, sobretudo, entre os assintomáticos [4].

Além disso, a política de controle populacional no Brasil sempre responsabilizou as mulheres pelo cuidado com os métodos de prevenção da gravidez e das DST, o que as fez utilizar massivamente as pílulas anticoncepcionais em detrimento do uso do preservativo, aumentando a vulnerabilidade à infecção pelo HIV, caracterizando o fenômeno de feminização da AIDS conforme segue o gráfico acima em virtude das relaçóes desiguais entre homens e mulheres [18].

Gráfico 2 - Proporção de casos de AIDS por faixa etária, Itabuna/BA, 2007 a 2010.

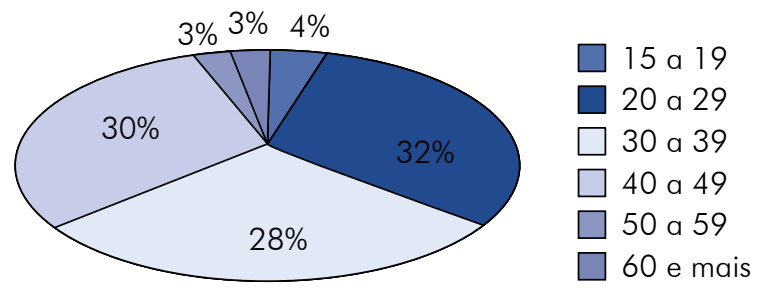

Quanto à faixa etária (Gráfico 2), verifica-se que o maior número de casos de AIDS concentra-se entre 20 e 29 anos (32\%), seguido por 40 e $49 \operatorname{anos}(30 \%)$ e 30 e 39 anos (28\%). Destaca-se ainda um aumento discreto no número de casos na faixa acima dos 50 anos, estimativa relacionada aos avanços tecnológicos com modificação no padrão sexual e comportamental dos sujeitos, a exemplo da utilização de próteses genitais, medicamentos para disfunção erétil, tratamentos hormonais continuados, o que tem contribuído para o prolongamento da vida sexual.

Outro fator de destaque seria a desmistificação do sexo por parte dos indivíduos de meia idade, tornando-os mais livres na condução das relaçóes sexuais. Contudo, existem questóes geracionais sobre prevençáo das DST que contribuem para o aumento da vulnerabilidade na terceira idade pelo fato dos mesmos carregarem tabus e mitos de geraçóes predecessoras, muitas vezes, internalizados nas relaçôes afetivas [19].

Verifica-se ainda que a prevalência nacional de AIDS encontra-se na faixa etária de 25 a 49 anos, o que valida os dados encontrados no estudo com maior probabilidade de infecção na população jovem. Estes dados justificam-se em virtude da concepção mais liberal sobre a sexualidade iniciada, no século $\mathrm{XX}$, com a realização de práticas sexuais múltiplas e menos comprometidas. Com isso, os jovens iniciaram as práticas sexuais precocemente, negligenciando o uso de preservativo e de métodos contraceptivos, aumentando a exposição ao HIV/ AIDS e demais DST [5,20]. Ademais, o Boletim Epidemiológico Ministerial aponta que os casos de AIDS no Nordeste encontram-se nas faixas etárias entre 40 e 49 anos e nos indivíduos com 60 anos e mais, dados similares aos achados no município de Itabuna [14].

Gráfico 3 - Proporção de casos de AIDS por escolaridade, Itabuna/BA, 2007 a 2010.

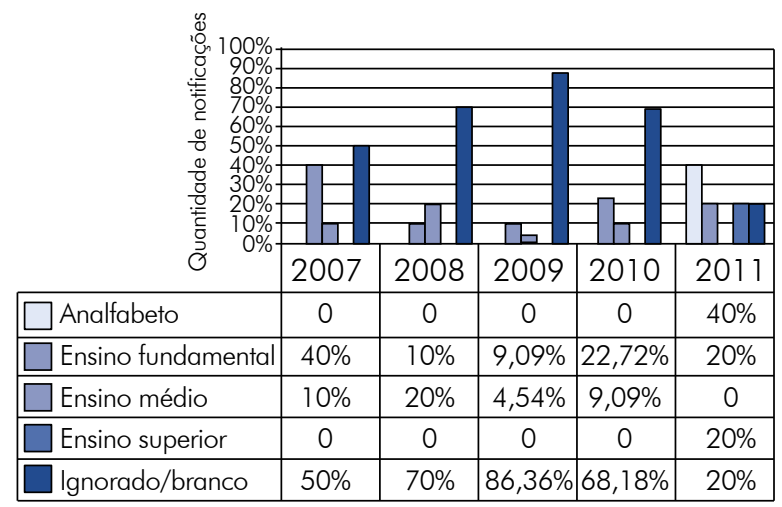

Quanto à variável escolaridade (Gráfico 3), observou-se maior proporção dos casos de AIDS em pessoas do ensino fundamental $(40 \% ; 10 \% ; 9,09 \%$; $22,72 \%)$, seguida do ensino médio (10\%; $20 \%$; $4,54 \% ; 9,09 \%)$ e aqueles com registro incompleto (50\%; 70\%; 86,36\%, 68,18\%) de 2007 a 2010. Percebe-se que os casos com registro incompleto mascaram a proporção real desta variável. $\mathrm{O}$ ano de 2011 credita maior parte dos casos de AIDS em analfabetos (40\%), no ensino fundamental (20\%), no ensino superior (20\%) e mantém ainda número de casos (20\%) como de notificação incorreta ou incompleta, apontando para a necessidade de melhorias no sistema de registro das DST por parte da equipe multidisciplinar.

Estudo realizado entre moradores de Sáo Paulo e Recife sobre DST revelou que o baixo nível de conhecimento sobre prevenção e transmissão de DST/AIDS associado à dificuldade de acesso aos serviços de saúde contribuem para o aumento no número de casos por ampliar a vulnerabilidade dos sujeitos a este agravo [21,22]. 
Gráfica 4 - Proporção de casos de AIDS por cor/etnia, Itabuna/BA, 2007 a 2010.

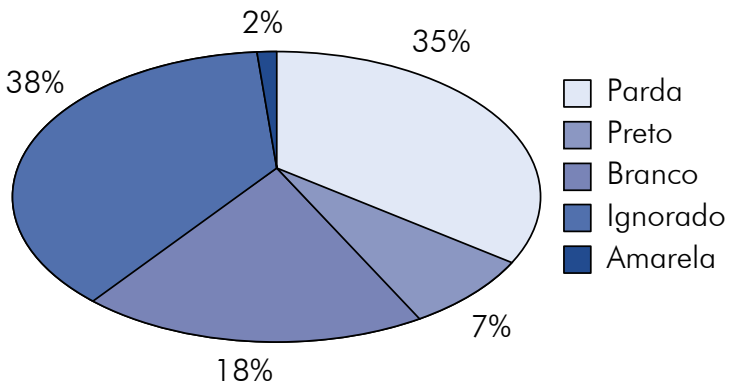

Com relação à cor e etnia (Gráfico 4), nota-se maior quantidade de casos entre indivíduos pardos (35\%), seguido de brancos (18\%) e pretos (7\%). Ressalta-se que ocorreu subnotificaçáo na variável cor/etnia com 38\% do total devido a não obrigatoriedade no preenchimento do campo. Segundo o IBGE, a cor parda, hoje, enquadra-se na etnia negra e, portanto, verifica-se uma grande proporção de casos de AIDS neste segmento populacional em decorrência da pauperização e menor condiçáo de acesso à saúde. $\mathrm{O}$ racismo é apontado como principal barreira social que limita a consolidação da prevenção e promoção à saúde [23].

\section{Hepatite B}

No que se refere à hepatite, foram registrados 470 casos, sendo 279 em homens e 191 em mulheres. Ao longo do tempo, a proporção de casos nos homens mostrou uma tendência mais ou menos regressiva, passando de 73,03\% (2007), 58,98\% (2008), 59,70\% (2009), 53,53\% (2010) e 57,40\% (2011). Em contraposição, houve um aumento no número de casos em mulheres ao longo dos cinco anos ao passar de 26,90\% em 2007 para $42,49 \%$ em 2011 conforme Gráfico 5.

Gráfico 5 - Proporção de casos de hepatite por faixa etária, Itabuna/BA, 2007 a 2010.

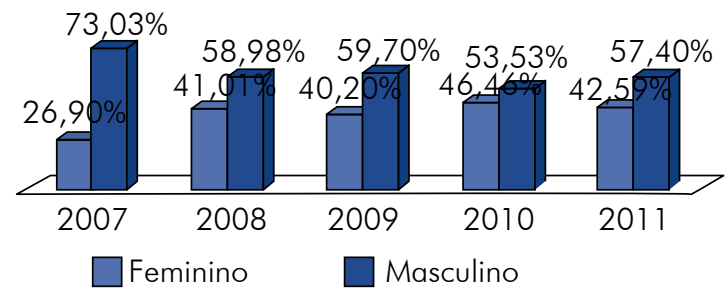

Nota-se ainda um maior número de casos de hepatite entre os homens quando comparado às mulheres. Tal fato relaciona-se à dificuldade dos homens em procurar os serviços de saúde com vistas à prevenção de doenças e promoção da saúde. Os mesmos costumam procurar pelos serviços em saúde quando manifestam sintomatologia o que amplia a progressão da doença.

Gráfico 6 - Proporção de casos de hepatite por faixa etária, ltabuna/BA, 2007 a 2010.

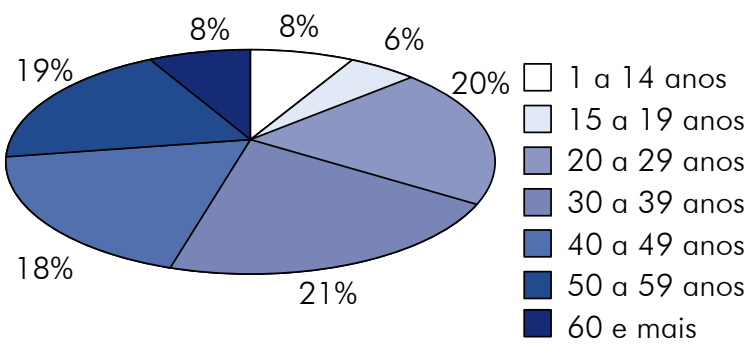

Analisando o número de casos de hepatite por idade (Gráfico 6), notou-se que a faixa etária mais acometida foi de 30 e 39 anos (21\%), 20 e 29 anos (20\%), 50 e 59 anos (19\%) e, 40 e 49 anos (18\%). Observa-se um aumento entre os idosos com 60 anos ou mais, apresentando uma proporção de $8 \%$ no período de cinco anos.

Estudos epidemiológicos mostraram distribuição similar de casos de hepatite por faixa etária, revelando significância estatística dos 30 e 39 anos, validando os dados do município de Itabuna. As taxas de incidência crescem, sobretudo entre mulheres de 50 e 59 anos, passando de 6,1 para 13,1 em uma série temporal de 10 anos. Isso acontece, em sua grande maioria, pela dificuldade em negociar o uso do preservativo nas relaçóes mais duradouras, por questóes geracionais e pela falta de informaçóes seguras sobre a prevenção $[22,24]$.

Apesar de os dados apontarem um aumento entre adultos jovens, percebe-se que a proporção cresce entre 60 anos ou mais. Ademais, estudos sobre prevalência das infecçóes pelo vírus das hepatites $\mathrm{B}$ e C em moradores de rua apontam para os achados neste estudo, e demonstram que a hepatite $\mathrm{C}$ contribui com $14 \%$ dos casos na faixa etária de 50 e 59 anos, seguido por 40 e 49 anos (10\%) e 30 e 39 anos (9,6\%). Por outro lado, a infecção pelo vírus $\mathrm{B}$ acometeu, preferencialmente, a faixa etária de 60 anos ou mais $(45,5 \%)$ e 50 e 59 anos $(41,9 \%)$ pelo fato de existir dificuldade para falar sobre a sexualidade e uso do preservativo. Tais concepçóes socialmente construídas dificultam o acesso aos meios de informação e prevenção. Soma-se ainda a dificuldade dos profissionais de saúde em abordar sobre o idoso sexuado, aquele que decide pelo próprio cuidado sexual $[25,26]$. 
Contudo, a proporção dos casos de hepatite entre 30 e 39 anos (21\%) em Itabuna, mostra-se discrepante dos dados apresentados pelo Ministério da Saúde, em que se verificou que o maior percentual de casos confirmados estava no grupo de 40 e 49 anos $(19,5 \%)$ e 50 e 59 anos $(54,4 \%)$, respectivamente. É possível entender que tais dados podem sugerir falhas na notificação desse agravo na faixa etária de 40 anos ou mais ou uma maior progressáo de casos na população jovem pela tendência às práticas sexuais desprotegidas, multiplicidade de parceiros, uso abusivo de álcool, experiências com drogas ilícitas e pressão negativa de seus pares $[27,28]$.

Gráfico 7 - Proporção de casos de hepatite por escolaridade, Itabuna/BA, 2007 a 2010

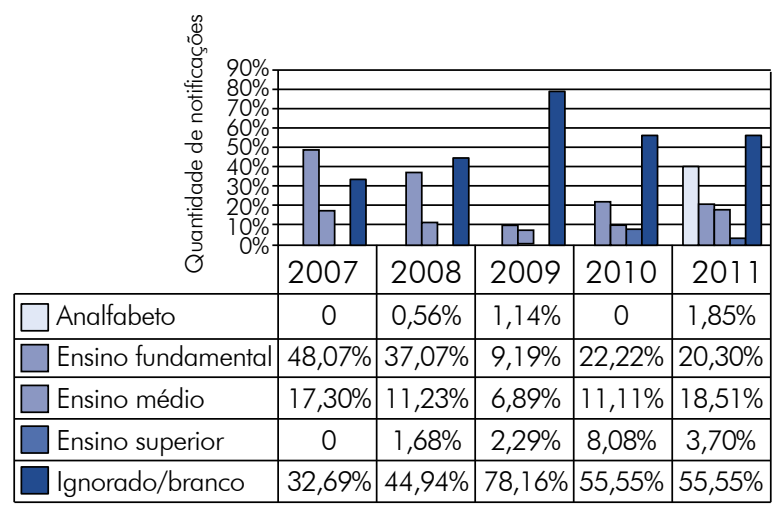

O Gráfico 7 demonstra a proporção de pessoas com hepatite segundo o nível de escolaridade. Verifica-se uma maior frequência em pessoas com ensino fundamental $(48,07 \%)$ e ensino médio $(18,51 \%)$. Percebe-se menor índice em indivíduos com nível de escolaridade no ensino superior, em torno de 8,08\%.

A maior proporção de notificaçóes para hepatite segundo a variável escolaridade definiu-se pelo ensino fundamental e médio. Esses dados convergem com os apresentados no Boletim Epidemiológico - Hepatites Virais, momento em que destacam que $57,4 \%$ dos casos acometidos possuíam em média de 4 e 11 anos de estudo [27].

Pesquisa realizada com adolescentes escolares de baixa renda sobre a vacinaçáo contra hepatite $B$ revela que o ambiente escolar torna-se o local para a aquisição de informaçóes e conhecimento para prevenção das DST. De acordo com o modelo ecológico comportamental, a família, a mídia, a história de punição, as influências culturais e de amigos e o próprio ambiente escolar influenciam na probabilidade do individuo adotar comportamentos de risco. Neste caso, a prevalência de indivíduos no ensino fundamental com hepatite pode estar atrelada a diversas falhas, incluindo aquelas ligadas ao processo educacional [28].

Gráfico 8 - Proporção de casos de hepatite por cor/ etnia, Itabuna/BA, 2007 a 2010

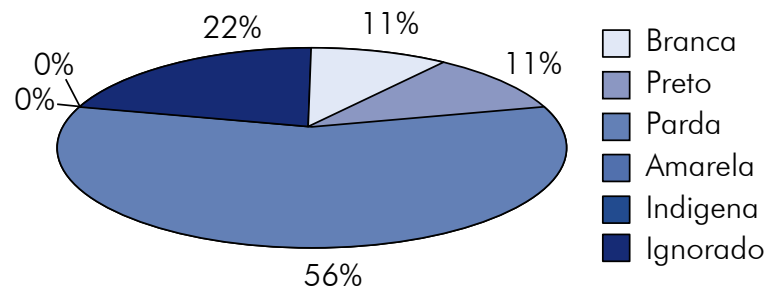

No Gráfico 8, observou-se que 56\% dos casos de hepatite aconteciam entre indivíduos de cor parda, seguido de $11 \%$ para a cor preta e branca, respectivamente. Nota-se uma proporção ampliada de casos na cor/etnia negra, ao somarmos pardos e pretos como determina o IBGE. Ademais, $22 \%$ dos casos notificados eram tidos como ignorados no que tange ao quesito cor/etnia em decorrência da náo preconizaçáo no preenchimento desta variável no campo da notificação.

Estudos epidemiológicos nacionais mostraram maior prevalência de hepatite $\mathrm{B}$ e $\mathrm{C}$ na cor branca, apresentando a seguinte distribuição de $75,7 \%$ e $84 \%$, respectivamente. A cor negra correspondeu com $13,1 \%$ e $8,5 \%$ e a etnia amarela com $9,7 \%$. Tal perfil nacional não se equipara ao estudo local. No município de Itabuna verifica-se predominância de hepatite em negros, fato explicado pela etnia majoritariamente negra no Estado da Bahia, decorrente dos processos históricos de colonização $[24,29]$.

\section{Conclusão}

A partir dos dados apresentados admite-se que o processo de notificação das principais DST em Itabuna ainda é incipiente. A grande proporção de variáveis ignoradas visualizadas na pesquisa, principalmente no quesito cor/etnia e escolaridade permitem identificar falhas e lacunas na coleta de informações epidemiológicas.

Diante do cenário exposto, estima-se que a frequência de DST seja maior do que revelam os dados oficiais, sugerindo elevada subnotificação de casos. Portanto, verifica-se a necessidade de implementar a notificação, alertando os profissionais de saúde sobre a importância destas variáveis para o desenvolvimento de estratégias de prevenção e controle das DST. Além disso, destaca-se a urgência em melhorar a qualidade dos dados coletados, bem como a realizaçáo de edu- 
cação continuada com os profissionais responsáveis pela organização e análise dos dados.

Contudo, apesar das fragilidades encontradas no SINAN percebe-se que o perfil epidemiológico das principais DST no município de Itabuna revela maior proporçáo de casos em homens quando comparado às mulheres, em indivíduos de cor negra, com faixa etária preponderante de 20 e 29 anos e com escolaridade básica.

Destaca-se a posição da mulher no que tange às DST por diversos motivos, dentre os quais: desigualdade de acesso aos direitos sexuais e reprodutivos, às características biológicas, ao preconceito e discriminação sofridos que dificultam a procura imediata aos serviços de saúde, sobretudo, aqueles relacionados à lesbianidade e bissexualidade, o desconhecimento sobre o próprio corpo, dificuldades na negociação no uso do preservativo e a possibilidade de violência sexual, ampliando a vulnerabilidade.

Assim, os profissionais de saúde devem atentar para a vulnerabilidade social, cultural econômica, política e sexual em que os sujeitos encontram-se imbuídos para traçar estratégias para a prevenção e controle das DST.

\section{Referências}

1. Ministério da Saúde. Manual de controle das doenças sexualmente transmissíveis. Brasília: MS; 2006.

2. Silva CM, Vargens OMC. A percepção de mulheres quanto à vulnerabilidade feminina para contrair DST/ AIDS. Rev Esc Enferm USP 2009;43(2):401-6.

3. Rocha LVS, Dantas VCD, Araújo JT, Rolim CK, Dantas AFR, Miranda HF et al. A vulnerabilidade às DST em regiāo com intensa prostituição e turismo sexual de Natal/RN. Rev Bras Anal Clin 2008;40(1):3-6.

4. Sousa LB, Barroso MGT. DST no âmbito da relação estável: análise cultural com base na perspectiva da mulher. Rev Enferm Esc Anna Nery 2009;13(1):123-30.

5. Ministério da Saúde. Epidemiologia: DST. Brasília: MS; 2011.

6. Murakami JK, Petrilli Filho JF, Telles Filho PCP. Conversando sobre sexualidade, IST e AIDS com adolescentes pobres. Rev Latinoam Enferm 2007;15(especial):1-3.

7. Maia C, Guilhem D, Freitas D. Vulnerabilidade ao HIV/AIDS de pessoas heterossexuais casadas ou em união estável. Rev Saúde Pública 2008;42(2):242-8.

8. Carneiro WS, Rodrigues JA, Felix MR, Athayde ACR, Lôbo KMS, Vilela VLR, et al. Percepçáo de vulnerabilidade feminina ao vírus da AIDS na estratégia de saúde da família. J Bras Doenças Sex Transm 2009;21(3):101-6.

9. Torres CA, Beserra EP, Barroso MGT. Relações de gênero e vulnerabilidade às doenças sexualmente transmissíveis: percepçóes sobre a sexualidade dos adolescentes. Rev Enferm Esc Anna Nery 2007;11(2):296-2.
10. Borba KP, Clapis MJ. Mulheres profissionais do sexo e a vulnerabilidade ao HIV/AIDS. J Bras Doenças Sex Transm 2006;18(4):254.

11. Araujo MAL, Silveira CB. Vivências de mulheres com diagnóstico de Doença Sexualmente Transmissível-DST. Rev Esc Enferm Anna Nery 2007;11(3):479-86.

12. Beserra EP, Pinheiro PNC, Alves MDS, Barroso MG. Adolescência e vulnerabilidade às doenças sexualmente transmissíveis: uma pesquisa documental. J Bras Doenças Sex Transm 2008;20(1):32-5.

13. Taquette SR. Interseccionalidade de gênero, classe e raça e vulnerabilidade de adolescentes negras às DST/ AIDS. Saúde e Sociedade 2010;19(2):51-62.

14. Ministério da Saúde. Boletim Epidemiológico AIDS. Brasília: MS; 2010.

15. Santos TS. Do artesanato intelectual ao contexto virtual: ferramentas metodológicas para a pesquisa social. Sociologias 2009;1(22):120-56.

16. Richardson RJ. Pesquisa social: métodos e técnicas. São Paulo (SP): Atlas; 1999.

17. Brasil. Lei no 9.610, de 19 de fevereiro de 1998. Dispóe sobre os direitos autorais. Brasília: MS; 1998.

18. Madureira VSF, Trentini M. Da utilização do preservativo masculino à prevenção de DST/AIDS. Rev Ciênc Saúde Coletiva 2008;13(6):1807-16.

19. Lazzarotto A. HIV/AIDS e meia idade: avaliação do conhecimento de indivíduos da regiáo do Vale do Sinos (RS), Brasil. Rev Ciênc Saúde Coletiva 2010;15(1):1185-90.

20. Falcáo Júnior JSP, Rabelo STO, Lopes EML, Freitas LV, Pinheiro AKB, Ximenes LB. Perfil e práticas sexuais de universitários da área de saúde. Rev Enferm Esc Anna Nery 2007;11(1):58-65.

21. Masson VA, Monteiro MA. Vulnerabilidade às Doenças Sexualmente Transmissíveis/AIDS e uso de drogas psicoativas por caminhoneiros. Rev Bras Enferm 2010;63(1):79-83.

22. Garcia S, Souza FM. Vulnerabilidades ao HIV/AIDS no Contexto Brasileiro: iniquidades de gênero, raça e geração. Rev Saude Soc 2010;19(2):9-20.

23. Spiassi AL, Faustino DM, Viso STR, Cavalheiro LO, Vichessi DFV, Sant'AnnaV, et al. O movimento negro do ABC Paulista: diálogos sobre a prevençáo das DST/ AIDS. Saúde e Sociedade 2010;19(2):122-33.

24. Cruz CRB, Shirassu MM, Martins WP. Comparação do perfil epidemiológico das hepatites $\mathrm{B}$ e $\mathrm{C}$ em um serviço público de São Paulo. Arq Gastroentero 2009;46(3):225-9.

25. Brito VCO, Parra D, Facchini R, Buchalla CM. Infecção pelo HIV, hepatites $\mathrm{B}$ e C e sífilis em moradores de rua, São Paulo. Rev Saúde Pública 2007;41(2):47-56.

26. Laroque MF, Affeldt AB, Cardoso DH, Souza GL, Santana MG, Lange CV. Sexualidade do idoso: comportamento para a prevenção de DST/AIDS. Rev. Gaúch Enferm 2011;32(4):774-80.

27. Ministério da Saúde. Boletim Epidemiológico - Hepatites Virais. Brasília: MS; 2011.

28. Oliveira MDS, Paggoto V, Matos MA, Kozlowski AG, Silva NR, Junqueira Neto AL, et al. Análise de fatores associados à não aceitaçáo da vacina contra hepatite $\mathrm{B}$ em adolescentes escolares de baixa renda. Rev Ciênc Saúde Coletiva 2007;12(5):1247-52.

29. Lopes F, Buchalla CM, Ayres JRCM. Mulheres negras e não-negras e vulnerabilidade ao HIV/AIDS no estado de São Paulo, Brasil. Rev Saúde Pública 2007;41(2):39-46. 